\title{
A Transmission Positron Microscope
}

T. Ootsuka, ${ }^{*}$ M. Inoue, ${ }^{*}$ M. Matsuya, ${ }^{*}$ T. Kurihara, ${ }^{* *}$ M. Doyama, ${ }^{* * * *}$ T. Oka, ${ }^{* * *}$ S. Jinno, ${ }^{* * *}$ and M. Fujinami***

* JEOL Ltd, 1-2 Musashino, 3-Chome, Akishima, Tokyo, 196-8558, Japan

** High Energy Accelerator Research Organization, 1-1 Oho, Tsukuba, Ibaraki, 305-0801, Japan

*** Department of Applied Chemistry, Chiba University, 1-33 Yayoi, Inage, Chiba, 263-8552, Japan

**** Teikyo University of Science and Technology, Uenohara, Yamanashi, 409-0913, Japan

A transmission positron microscope (TPM) JEM-1011B has been newly developed to survey the difference of the transmission images by positron $\left(\mathrm{e}^{+}\right)$and electron $\left(\mathrm{e}^{-}\right)$[1]. Both micrographs up to a few thousand magnification and diffraction patterns are presented for $\mathrm{e}^{+}$and $\mathrm{e}^{-}$beams. Fig. 1 shows the brightness enhancer (BE) [2] and the TPM system. The BE accepts the $\mathrm{e}^{+}$beam generated in the $\mathrm{e}^{+}$ facility [3] with an intensity $10^{7} \mathrm{e}^{+} / \mathrm{s}$ which is $10-100$ times larger than that obtained by commercial radioisotopes. The beam (up to $30 \mathrm{keV}$ ) from BE is guided to the TPM by magnetic relay lenses placed in the differential pumping system along the linear optical axis. The $\mathrm{e}^{-}$beam is focused using a sector magnet on the same crossover point as the $\mathrm{e}^{+}$beam. This method does not decrease the enhanced brightness of the $\mathrm{e}^{+}$beam. The beams can be monitored by several micro-channel plates (MCP) which enable the axis alignment of the weak beams. The final image is monitored and recorded by the MCP with an image processing system. The transmission micrographs for various materials have been taken in several diameters of objective aperture. Micrographs of a $\mathrm{Au}(100)$ film with $10 \mathrm{~nm}$ thickness and the diffraction patterns from the same area are shown in Figs 2 and 3, respectively. The micrograph by $\mathrm{e}^{+}$in Fig.2(A) is much better quality than that reported by the previous investigators [4]. The diffraction patterns by $\mathrm{e}^{+}$are comparable to those by $\mathrm{e}^{-}$. These features make it possible to compare the small difference between two beams. The results show that the transmittance of the Au film for $\mathrm{e}^{+}$is slightly larger than that for $\mathrm{e}^{-}$.

[1] M. Doyama et al (Panelists), Positron Annihilation, ICPA-7, World Scientific Co., (1985) p437

[2] M. Fujinami, S. Jinno, M. Fukuzumi, T. Kawaguchi, K. Oguma et al, Anal. Sci., 24 (2008) 73

[3] T. Kurihara, Y. Nagashima, T. Sidara, H. nakajima et al, Mater. Sci. Forum, 445-446 (2004) 486

[4] J.V. House and A. Rich, Phys. Rev. Lett., 60 (1988) 169

[5] This work was partially supported by a grant from the "System Development Program for Advanced Measurement and Analysis" of the Japan Science and Technology Agency (JST). We thank R. Suzuki, T. Ohdaira, Y. Kobayashi, N. Oshima, A. Uedono, T. Akahane, X. Cao, Y. Kogure, M. Inoue, K. Suzuki, Z. Ohyama and T. Miyokawa for fruitful discussions. 


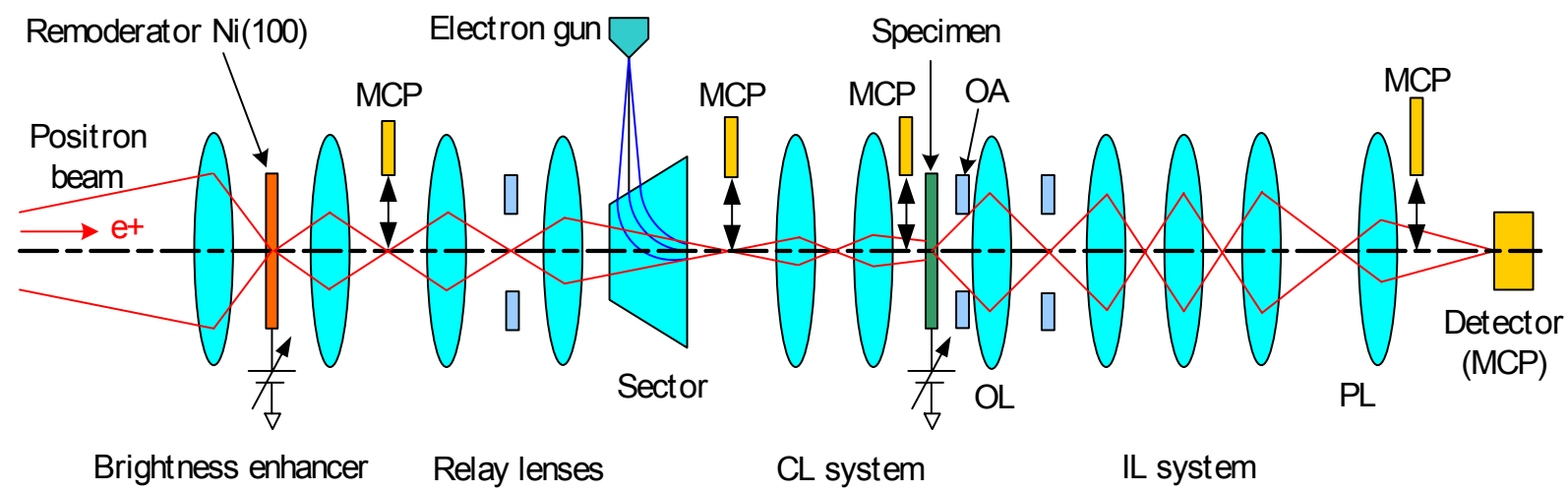

Fig.1. Schematic illustration of positron and electron beam optics.
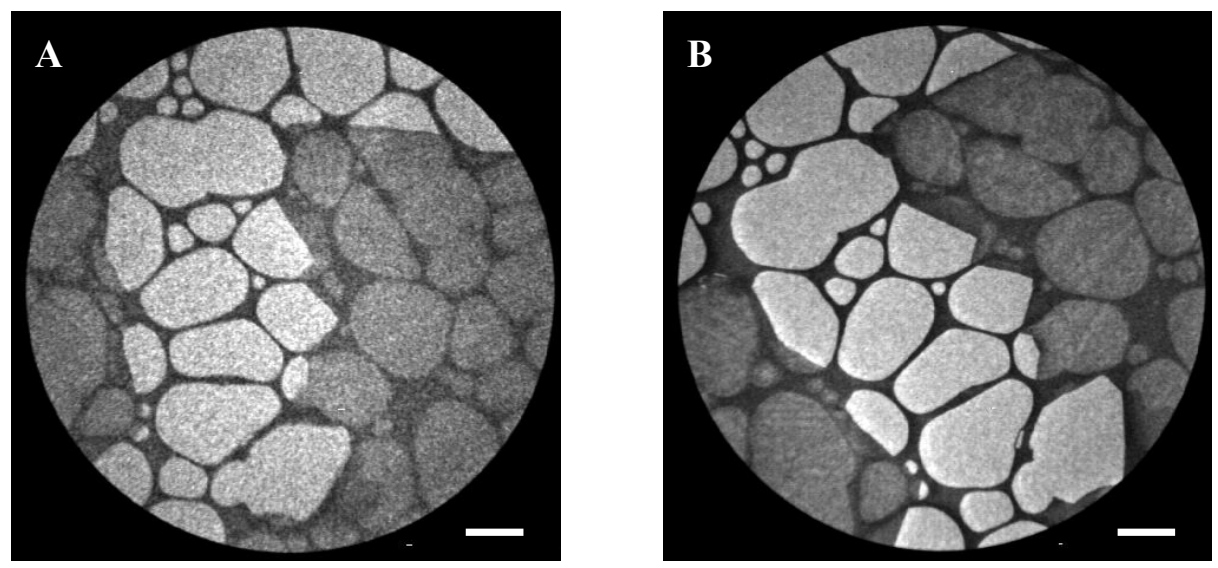

Fig. 2. Transmission micrographs of a $\mathrm{Au}$ (100) film using $30 \mathrm{keV}$ beams.

A. Positron. B. Electron. Scale bar $=4 \mu \mathrm{m}$. The film is mounted on a micro-grid.
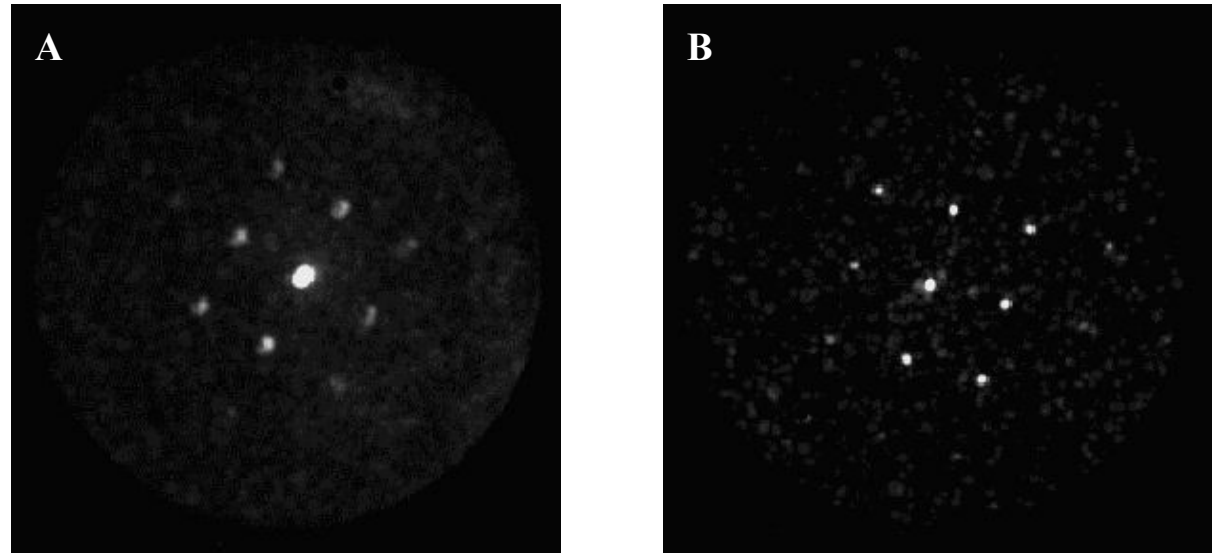

Fig. 3. Diffraction patterns indicating $\{002\}$ and $\{022\}$ for the $\mathrm{Au}(100)$ film using $30 \mathrm{keV}$ beams. A. Positron. B. Electron. 